REVUE DE L'INSTITUT

FRANÇAIS D'HISTOIRE

EN ALLEMAGNE

\section{Revue de l'IFHA}

Revue de l'Institut français d'histoire en Allemagne

$6 \mid 2014$

IFHA 6

\title{
L'Exzellenzcluster 1027 : « Laboratoire interdisciplinaire "image, savoir et création" »
}

Université Humboldt de Berlin

\section{Bérénice Zunino}

\section{OpenEdition}

\section{Journals}

Édition électronique

URL : http://journals.openedition.org/ifha/8023

DOI : 10.4000/ifha. 8023

ISSN : 2198-8943

Éditeur

IFRA - Institut franco-allemand (sciences historiques et sociales)

Édition imprimée

Date de publication : 31 décembre 2014

ISSN : 2190-0078

Référence électronique

Bérénice Zunino, «L'Exzellenzcluster 1027 : « Laboratoire interdisciplinaire "image, savoir et création" » », Revue de l'IFHA [En ligne], 6 | 2014, mis en ligne le 31 décembre 2014, consulté le 03 mai 2019. URL http://journals.openedition.org/ifha/8023 ; DOI : 10.4000/ifha.8023

Ce document a été généré automatiquement le 3 mai 2019.

(CIFHA 


\title{
L'Exzellenzcluster 1027 : « Laboratoire interdisciplinaire "image, savoir et création" "
}

\author{
Université Humboldt de Berlin
}

\author{
Bérénice Zunino
}

https://www.interdisciplinary-laboratory.hu-berlin.de Responsables : Horst Bredekamp et Wolfgang Schäffner

1 Porté par l'université Humboldt de Berlin, ce cluster d'excellence a été créé en 2012. Il part du postulat que la science est une création. Le savoir a depuis toujours été créé par l'architecture, les outils et les modèles, les moyens d'informations et en particulier les images. En raison du développement des techniques de production numériques de l'image, l'importance de la création dans la fabrication et la perception du savoir a changé de nature depuis une cinquantaine d'années. Instruments de modélisation et de transmission ou encore outils de démonstration et d'archivage, les images ont engendré un changement profond tant dans les sciences humaines et sociales et les sciences exactes que dans les domaines de la technique et de la médecine. Elles permettent d'appréhender des systèmes de données complexes et presque infinis. Loin de posséder seulement une dimension immatérielle, elles créent un lien entre les ressources numériques et leur contenu et forment ainsi un vaste fonds de savoirs diversifiés. Elles décloisonnent de ce fait les disciplines.

2 Fort de ce constat, le cluster d'excellence 1027 s'appuie, dans une perspective résolument interdisciplinaire, sur la notion de création, considérée comme un paradigme des procédés modernes de conception et de production, pour étudier les images et le savoir. Ces deux objets d'étude sont conçus comme des processus de création communs à tous les domaines scientifiques. Vingt-deux disciplines, dont l'archéologie, la littérature, l'histoire de l'art, l'architecture, l'ethnologie, la médecine, la psychologie, la biologie ou encore l'informatique, sont représentées par de nombreuses institutions de recherche universitaires et extra-universitaires telles que l'Université libre, l'Université technique, 
l'Université des arts de Berlin, le Musée des arts décoratifs, le Musée d'histoire naturelle de Berlin et le Musée de l'histoire de l'hygiène de Dresde.

Ce projet est divisé en cinq groupes de recherche. Réunis thématiquement sous l'intitulé " divergences méthodologiques », les trois premiers axes confrontent les approches et les méthodes d'analyse fondamentalement différentes des domaines d'étude divers pour tenter d'en dégager des pratiques communes, d'en proposer une synthèse novatrice et de créer des interactions nouvelles entre les disciplines. Le premier axe, intitulé "historicisation et expérimentation", s'est donné pour objectif de croiser les deux grandes méthodes traditionnellement induites par les sciences humaines et sociales, d'une part, et les sciences exactes, d'autre part, en les appliquant respectivement à l'autre culture scientifique. Cette hybridation des méthodes devrait permettre l'émergence de nouveaux modes d'analyse. Dans une démarche similaire, le deuxième axe, " analyse et synthèse ", est consacré à la comparaison des deux grandes méthodes qui caractérisent les sciences historiques et les sciences de la technique afin de créer de nouvelles interactions, tandis que dans le troisième axe, " matérialité et virtualité », une attention particulière est accordée aux savoirs qui s'agrègent à partir d'images, de modèles et de structures.

Parallèlement à cette réflexion théorique sur les divergences méthodologiques inhérentes aux différentes disciplines, un quatrième axe se propose d'analyser les « convergences novatrices » à partir d'études de cas. Pour ce faire, cinq thématiques ont été retenues. Outre les modèles et les échelles (1), les structures, les réseaux et les superficies (2), la mobilité (3) et les éléments de la conception (4), la visualité (5) occupe une place centrale. Dans cette perspective, l'historien de l'art et spécialiste de l'image Horst Bredekamp, célèbre pour son essai sur l'« acte imagé »1, et le linguiste Jürgen Trabant postulent un pouvoir agissant des images. Qu'elles relèvent de l'histoire de l'art, de l'informatique ou encore de l'imagerie médicale, ces dernières sont considérées comme les agents de processus historiques et scientifiques. Les résultats des recherches de ce quatrième axe donneront lieu à des manifestations et à des expositions qui rendront accessibles à un large public les synergies dégagées.

Enfin le cinquième axe est conçu comme un lieu d'autoréflexion sur les approches du cluster d'excellence. Fondé sur l'interdisciplinarité, il incite à une mise à distance critique des objets d'étude, des formes et des lieux de travail du groupe de recherche dans son ensemble.

6 Parmi les nombreuses publications des membres de ce cluster d'excellence qui ont servi de base à la réflexion, on peut citer Denken in Bildern. 31 Positionen zu Kunst, Museum und Wissenschaft, édité par Moritz Wullen et Günther Schauerte (Ostfildern : H. Cantz, 2008), l'article de Susanne Muth, « Gewalt im Bild. Das Phänomen der medialen Gewalt im Athen des 6. und 5. Jh. v. Chr.", paru dans la revue ICON ( ${ }^{\circ} 1$ 1, 2008), Grand Paris - Métropole Douce. Propositions for the Post-Kyoto Metropolis, édité par Finn Geipel et l'équipe LIN (Paris : J.M. Place, 2009), l'ouvrage de Horst Bredekamp, Theorie des Bildaktes (Berlin : Suhrkamp, 2010) et celui de Sigrid Weigel, Grammatologie der Bilder (Berlin : Suhrkamp, 2012). 


\section{NOTES}

1. Horst Bredekamp, Theorie des Bildakts, Berlin : Suhrkamp, 2010.

\section{AUTEUR}

BÉRÉNICE ZUNINO

(Paris-Sorbonne/IFHA) 